\title{
correspondence
}

\section{Europe versus itself}

SIR,-With reference to the editorial "Million-dollar problem-billion dollar solution?" (14 July, page 89), the problem of sulphur pollution is not a problem of Norway versus the United Kingdom, but rather of Europe versus itself.

The OECD study, to which the article refers, has shown that there is a more or less continuous export and import of pollutants between European countries and that the problem is not confined to any two countries. Nor can it be solved by individual national control programmes. It should also be emphasised that the problem is not predominantly confined to the effects of acid rainfall on fish populations. The effects of sulphur pollution fall into four main areas: human health, vegetation, the fresh water ecology, and corrosion of metals, painted surfaces and other materials.

It is established that several categories of human disease are linked to the occurrence of sulphur dioxide and associated pollutants in the atmosphere. Governments' efforts to improve the quality of the air so as to provide better health protection are likely to have the reduction of sulphur emissions as one of their main targets.

With regard to corrosion we know that material damage due to sulphur pollution in Sweden and the United States cost between $\$ 5$ and $\$ 10$ per capita per year in the early 1970s. We know that damage is inflicted on vegetation in areas polluted with high concentrations of sulphur. It is also suspected that the dispersion of sulphur will result in decreased tree growth in areas with relatively low average concentrations of pollutant. The economic consequences of such effects can be highly significant.

We must also expect synergetic or combined effects in wide areas within Europe where various harmful pollutants are dispersed through the atmosphere. Efforts should now be directed at developing a coordinated European policy for reducing emissions. The costs involved will, it is true, be formidable. Only gradual improvement can therefore be expected. The result, however, will be beneficial to health, to materials susceptible to corrosion and to the quality of the natural environ-

ment all over Europe, with particular benefits accruing to people and areas closest to emission sources.

Yours faithfully,

ERIK LYKKe

Ministry of Environment,

Norway

\section{Nutrition in food policies}

Sir,-Blythe and Rush (4 August, page 386) outlined the state of the cautious dialectics of a UK food policy which incorporates nutritional criteria. The arguments are complex and controversial, not least because of the range of disciplinary interests involved. Three of the arguments referred to by the authors warrant further comment.

First, is the search for 'absolute proof' of causal linkages between dietary constituents and diseases necessary before recommendations commensurate with formulating food policy can be made? Is 'absolute proof' actually attainable? The scientific method sets out to falsify theories through experimentation, never to prove their validity, it is neither the intent of the method nor logically possible to prove the validity of theories, irrespective of the number of experiments. Thus, the argument should be concerned with the degree of confidence that can be attached to the corroboration between theories and observations in scientific experiments. Thus, for example, where there exists a sustainable conjuncture that a particular level of dietary fat (or saturated fat) is detrimental to health, this should be sufficient basis for a recommendation.

Second, the lack of an explicit food policy which links nutrition and health to food supply is tantamount to accepting, by default, that the fragmentary components of existing policies which affect food consumption adequately accommodate nutrition and health goals. Equally, the nutrition and medical professions, by failing to make recommendations, are by default condoning the present dietary trends.

Concerns that the introduction of nutritional goals into agriculture and food supply policy would require significant structural changes are exaggerated. The food supply industry is not static: at present there is a programme for expansion of UK agriculture, and for further structural change within the EEC. There is no reason to believe that the changes needed to include nutritional goals would be any greater than those already under discussion or being implemented for sectional economic interests alone.

Thirdly, the controversy surrounding state intervention in consumers' freedom of choice through a food and nutrition policy is a red herring. The extent to which a real freedom of choice exists is academic, but it is evident that consumers' choice is directed by a wide range of measures from diverse sources. Effectively, consumers are free to choose from numerous, but pre-determined, alternatives, and that choice is further constrained by such factors as their income, access to information, and susceptibility to advertising. A food and nutrition policy would not necessarily impose further restrictions on choice, but could provide the consumer with a more rational and coherent basis for his choice. This implies that all consumers have access to a nutritionally adequate diet with established health safeguards, while maintaining the widest feasible variety and availability of foods. As a wide access to relevant information on nutrition, health and foods is a component of most conceptions of a food and nutrition policy, it can be argued that such a possibility would increase the consumer's capacity to exercise a free choice, rather than reduce it.

Yours faithfully,

C. J. Robins

Reading,

Berkshire

\section{Crater good}

SIR,-I shall say an Ave Mare for the soul of David W. Hughes (15 September, page 197).

Yours faithfully,

Leicestershire, UK

L. ROSE

\section{Correction}

In T. R. C. Boyde's quoted derivation of the word 'enzyme' (15 September, page 194), the Greek for 'in yeast' should have read $\varepsilon v \zeta v \mu \eta_{1}$ not $\varepsilon \nu \xi v \mu \eta$. The modern Greek would read $\varepsilon \nu \zeta v \mu o \sigma$. 